\title{
Hypocretin/orexin prevents recovery from sickness
}

\author{
SUSUMU TANAKA ${ }^{1,2}$, HIROMI TOYODA $^{1,3}$, YOSHIKO HONDA $^{1}$, YASUKO SEKI $^{1}$, \\ TAKESHI SAKURAI ${ }^{4,5}$, KAZUKI HONDA ${ }^{1}$ and TOHRU KODAMA ${ }^{1}$
}

\begin{abstract}
${ }^{1}$ Sleep Disorders Project, Department of Psychiatry and Behavioral Science, Tokyo Metropolitan Institute of Medical Science, Tokyo 156-8506; ${ }^{2}$ Department of Anatomy and Cell Science, Kansai Medical University, Hirakata, Osaka 573-1010,

${ }^{3}$ Department of Human Genetics, Graduate School of Medicine, The University of Tokyo, Tokyo 113-0033;

${ }^{4}$ Department of Molecular Neuroscience and Integrative Physiology, Faculty of Medicine, Kanazawa University, Kanazawa, Ishikawa 920-8640; ${ }^{5}$ International Institute for Integrative Sleep Medicine,

University of Tsukuba, Tsukuba, Ibaraki 305-8577, Japan
\end{abstract}

Received March 13, 2015; Accepted June 18, 2015

DOI: $10.3892 /$ br.2015.491

\begin{abstract}
Sickness behavior is defined as states of lethargy, depression, anxiety, loss of appetite, hypersomnia, hyperalgesia, reduction of grooming and failure to concentrate that can be induced by inflammatory diseases, such as infections and cancer. Recent findings revealed that the lipopolysaccharide (LPS) injection causes lethargy as a consequence of the inhibition of hypocretin signaling. The hypocretin system maintains the vigilance state in various physiological processes. In order to investigate the sleep arousal system against sickness behavior, LPS-induced sickness behavior was examined in hypocretin-ataxin-3 transgenic mice, whose hypocretin neurons were postnatally ablated. Sleep-wake activity was determined following the administration of LPS at Zeitgeber time (ZT) 8.0 in ataxin-3 transgenic mice, and the age-, gender-matched wild-type littermates. LPS injection induced increases in non-rapid eye movement (REM) sleep in the matched wild-type littermates. In addition, a further increase in periods of sleep according to the loss of hypocretin neurons was identified in the ataxin-3 transgenic mice. A marked reduction of awakening during ZT12-ZT18 was observed as expected following LPS injection in the mouse lines. The increase in the period of non-REM sleep was not observed on the next day following LPS administration in either of the mouse lines. Complete recovery of physical activity was not observed in the matched wild-type littermates. Ataxin-3 transgenic mice recovered their physical activity to the same level as that on the first day before LPS administration. These results suggest the possibility that a faster recovery is the result of deeper resting according to
\end{abstract}

Correspondence to: Dr Susumu Tanaka, Department of Anatomy and Cell Science, Kansai Medical University, 2-5-1 Shinmachi, Hirakata, Osaka 573-1010, Japan

E-mail: tanakass@hirakata.kmu.ac.jp

Key words: sleep, wakefulness, sickness behaviour, hypocretin, orexin the absence of hypocretin neurons, as ataxin-3 transgenic mice demonstrated more non-REM sleep.

\section{Introduction}

Sickness behavior is characterized as a reduction of normal locomotor activities in response to circadian rhythms and psychological changes that develop in inflammatory diseases, including infections and cancers. Administration of the bacterial endotoxin lipopolysaccharide (LPS) induces sickness behavior in rodents. Recently, hypocretin (also known as orexin) signaling was identified as one of the neural mediators against sickness behavior (1). Grossberg et al (1), showed that LPS injection induced the suppression of normal hypocretin signaling and inactivity. The hypothalamic neuropeptide hypocretin regulates various physiological processes such as wakefulness, sleep, food intake, energy expenditure and reward $(2,3)$. Expression changes in the hypothalamic hypocretin have been reported regarding food restriction (4) and other physiological changes (5) to maintain the vigilance state. However, there is no study of how the absence of hypocretin is affected against sickness behavior.

In the present study, the significance of hypocretin signaling against sickness behavior was examined using LPS-induced inflammation in hypocretin-ataxin-3 transgenic mice, whose hypocretin neurons were postnatally ablated (6). In addition, the present study is important for the elucidation of the mechanism in narcolepsy patients with inflammatory diseases, whose hypocretin neurons were ablated (7).

\section{Materials and methods}

Ethical statement. All the experiments were conducted in accordance with the 'Guidelines for Care and Use of Laboratory Animals' of the National Institutes of Health and were approved by the Ethics Committee on Animal Experiments of Tokyo Metropolitan Institute of Medical Science (Tokyo, Japan).

Animals. The presence of the ataxin-3 transgene was identified by polymerase chain reaction using tail DNA. Male 
A

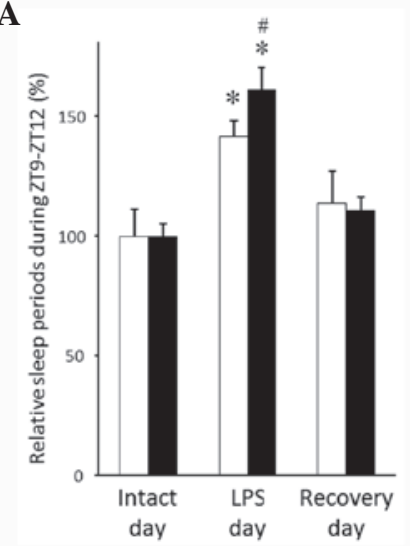

B

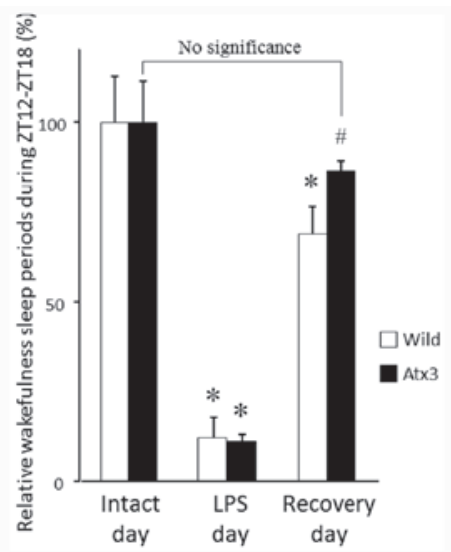

Figure 1. Changes in sleep/wakefulness periods following lipopolysaccharide (LPS) injection in ataxin-3 transgenic mice. (A) Relative sleep period during Zeitgeber time 9 (ZT9)-ZT12 standardized by the intact day. (B) Relative wakefulness periods during ZT12-ZT18 standardized by the intact day. "P<0.05, significant differences in each day compared with the intact day. ${ }^{~} \mathrm{P}<0.05$, significant differences in ataxin-3 transgenic mice compared with wild-type littermates. White bars indicate the matched wild-type littermates $(\mathrm{n}=4)$ and black bars are ataxin-3 transgenic mice $(\mathrm{n}=4)$. Atx 3 , ataxin-3 transgenic mice; wild, wild-type littermates.

mice (12-week-old) were anesthetized and implanted with devices for continuous monitoring of electroencephalography (EEG)/electromyography (EMG), as described previously (8). Mice were housed under a 12-h light/dark cycle with lights on from 8:00 a.m. to 8:00 p.m., corresponding to Zeitgeber time (ZT) $0-12 \mathrm{~h}$ at $22-24^{\circ} \mathrm{C}$, with ad libitum access to food and water. Mice were allowed to habituate to the recording conditions for 2 weeks.

LPS injections. On the second day at ZT8, mice received intraperitoneal injections of either LPS (Sigma-Aldrich, St. Louis, MO, USA) $(250 \mu \mathrm{g} / \mathrm{kg})$ dissolved in $0.9 \%$ saline or $0.9 \%$ saline alone. Each mouse was recorded for 3 consecutive 24-h periods. EEG/EMG were recorded without injection on the first day (intact day), and mice received LPS or saline alone and underwent EEG/EMG on the second day (LPS or saline day, respectively), and subsequently EEG/EMG were recorded without injection on the third day again (recovery day). A total of 4 male ataxin-3 transgenic mice and 4 matched wild-type littermates were recorded concurrently. EEG/EMG signals were amplified using an EEG/EMG amplifier (MEG-6116; Nihon Kohden Corp., Tokyo, Japan) through a 5-strand cable with a slip-ring that allowed free movement of the mice. The amplifier was connected to a personal computer with an analog-to-digital converter and SleepSign software (Kissei Comtec, Nagano, Japan) for acquiring and processing data. EEG/EMG records were visually scored into 4-sec epochs of wakefulness (high EMG amplitude), rapid eye movement (REM) sleep [silent low EMG amplitude, low EEG amplitude with high values in the $\theta$ band (4.0-8.0 Hz)], and non-REM sleep [low EMG amplitude, high EEG amplitude with high power density in the $\delta$ band $(0.5-4.0 \mathrm{~Hz})]$ according to the standard criteria of rodent sleep (9). Relative sleep and wakefulness periods were standardized by the mean of each proportion of the corresponding time on the intact day. The standardized data are expressed as the mean of 4 independent animals \pm standard error.

Statistical analysis. Distributions of sleep/wakefulness periods in ataxin-3 transgenic mice were compared to those in the matched wild-type littermates using Student's t-test (two-tailed test). $\mathrm{P}<0.05$ was considered to indicate a statistically significant difference.

\section{Results}

Changes in sleep/wakefulness periods. There was no significant difference regarding non-REM sleep periods in the light phase following injection (ZT9-ZT12) or wakefulness periods in the dark phase (ZT12-ZT18) between the intact day and saline day in ataxin-3 transgenic mice or matched wild-type littermates, respectively (data not shown).

By contrast, non-REM sleep periods (ZT9-ZT12) were significantly increased following LPS injection on the LPS day in ataxin-3 transgenic mice $(\mathrm{P}<0.01)$ or matched wild-type littermates $(\mathrm{P}<0.0005)$ compared with mice on the intact day (Fig. 1A). Non-REM sleep periods during ZT9-ZT12 of the ataxin-3 transgenic mice were significantly increased following LPS injection on the LPS day compared with that of the matched wild-type littermates $(\mathrm{P}<0.05)$ (Fig. 1A), suggesting that the ataxin-3 transgenic mice demonstrate more sleep during sickness behavior. Wakefulness periods during ZT12-ZT18 were significantly decreased following LPS injection on the LPS day in ataxin- 3 transgenic mice $(\mathrm{P}<0.0005)$ and matched wild-type littermates $(\mathrm{P}<0.0005)$ compared with mice on the intact day (Fig. 1B). On the day following LPS injection (recovery day), the significant reduction in the wakefulness period during ZT12-ZT18 was retained in the matched wild-type littermates $(\mathrm{P}<0.05)$; however, there was no significant difference between wakefulness periods on the intact day or recovery day in the ataxin-3 transgenic mice compared with mice on the intact day (Fig. 1B).

\section{Discussion}

Previously, LPS injection was reported to induce increases in non-REM sleep (10). In the present study, increases in the periods of non-REM sleep induced by LPS injection were observed in the matched wild-type littermates. In addition, further increases in sleep according to hypocretin loss in 
ataxin-3 transgenic mice were demonstrated. The marked reduction of activity was observed as expected following LPS injection in the mouse lines. The marked increase in the quantity of sleep was not observed on the recovery day in either line. The complete recovery of the physical activity was not observed in the matched wild-type littermates; however, it was confirmed that the ataxin-3 transgenic mice recovered their physical activity to the same as the intact day levels.

Hypocretin is known to regulate the sleep arousal process. When there are no hypocretin neurons, it can be hypothesized that the ataxin-3 transgenic mice will show an increase in sleep. Hypocretin is suppressed in wild-type mice by inflammation (1); however, it is believed that hypocretin neurons are not completely inhibited in LPS-induced sickness behavior of wild-type mice. Therefore, differences in sickness behavior between ataxin-3 transgenic mice and matched wild-type littermates on the LPS day in the present study may be explained with/without hypocretin signaling.

Notably, it was suggested that the recovery from sickness in the ataxin-3 transgenic mice was quicker when compared with that in the matched wild-type littermates. As the ataxin-3 transgenic mice get more sleep, it is suggested that their faster recovery is the result of longer rest on the LPS day.

Hypocretin is necessary for healthy people to maintain wakefulness. Hypocretin, however, may be one of the factors preventing recovery during sickness. The evolutionary merit of this system is explained by wild animals, for whom an extended, deep sleep may be dangerous. By contrast, humans are usually completely protected when sick. Therefore, recovery during a cold may be faster if combination therapies involving cold medicine and a hypocretin antagonist $(11,12)$ are administered.

\section{Acknowledgements}

The present study was supported by JSPS KAKENHI grant no. 23700458 to S. Tanaka.

\section{References}

1. Grossberg AJ, Zhu X, Leinninger GM, Levasseur PR, Braun TP Myers MG Jr and Marks DL: Inflammation-induced lethargy is mediated by suppression of orexin neuron activity. J Neurosci 31: 11376-11386, 2011

2. de Lecea L and Sutcliffe JG: The hypocretins and sleep. FEBS J 272: 5675-5688, 2005.

3. Sakurai T: The neural circuit of orexin (hypocretin): Maintaining sleep and wakefulness. Nat Rev Neurosci 8: 171-181, 2007.

4. Mieda M, Willie JT, Hara J, Sinton CM, Sakurai T and Yanagisawa M: Orexin peptides prevent cataplexy and improve wakefulness in an orexin neuron-ablated model of narcolepsy in mice. Proc Natl Acad Sci USA 101: 4649-4654, 2004.

5. Harris GC and Aston-Jones G: Arousal and reward: A dichotomy in orexin function. Trends Neurosci 29: 571-577, 2006.

6. Hara J, Beuckmann CT, Nambu T, et al: Genetic ablation of orexin neurons in mice results in narcolepsy, hypophagia and obesity. Neuron 30: 345-354, 2001.

7. Peyron C, Faraco J, Rogers W, et al: A mutation in a case of early onset narcolepsy and a generalized absence of hypocretin peptides in human narcoleptic brains. Nat Med 6: 991-997, 2000.

8. Chemelli RM, Willie JT, Sinton CM, et al: Narcolepsy in orexin knockout mice: Molecular genetics of sleep regulation. Cell 98: 437-451, 1999.

9. Radulovacki M, Virus RM, Djuricic-Nedelson M and Green RD: Adenosine analogs and sleep in rats. J Pharmacol Exp Ther 228: 268-274, 1984.

10. Morrow JD and Opp MR: Diurnal variation of lipopolysaccharide-induced alterations in sleep and body temperature of interleukin-6-deficient mice. Brain Behav Immun 19: 40-51, 2005.

11. Cao $\mathrm{M}$ and Guilleminault C: Hypocretin and its emerging role as a target for treatment of sleep disorders. Curr Neurol Neurosci Rep 11: 227-234, 2011.

12. Roecker AJ and Coleman PJ: Orexin receptor antagonists: Medicinal chemistry and therapeutic potential. Curr Top Med Chem 8: 977-987, 2008. 